\title{
3 Research Square

\section{Giant Intrapericardial Thymic Carcinoma Invading Superior Vena Cava and Right Atrium: A Case Report}

\section{Kai Zhu}

Zhongshan Hospital Fudan University

\section{Weijie Chen}

Zhongshan Hospital Fudan University

Guobing Liu

Zhongshan Hospital Fudan University

Yun Zhao

Zhongshan Hospital Fudan University

\section{Hongqiang Zhang}

Zhongshan Hospital Fudan University

\section{Sun Pan}

Zhongshan Hospital Fudan University

Chunsheng Wang ( $\nabla$ wangchunsheng@fudan.edu.cn )

Zhongshan Hospital Fudan University https://orcid.org/0000-0001-7774-6191

\section{Case report}

Keywords: Thymic carcinoma, Intrapericardium, Multimodality imaging

Posted Date: September 1st, 2020

DOI: https://doi.org/10.21203/rs.3.rs-63638/v1

License: (c) (1) This work is licensed under a Creative Commons Attribution 4.0 International License. Read Full License 


\section{Abstract}

Background: Thymic carcinoma is an epithelial malignant tumour that rarely presents in the pericardial cavity.

Case presentation: Herein, we describe a patient who suffered giant intrapericardial mass with infiltration of the superior vena cava (SVC) and right atrium (RA). The mass was evaluated by multimodality imaging. Surgeons performed en bloc resection of mass and reconstruction of SVC and RA. Pathological results supported a diagnosis of thymic carcinoma.

Conclusions: To our knowledge, this was the largest pericardial thymic carcinoma ever to be reported in the literature. This case demonstrates the utility of multimodality imaging in evaluating the pericardial mass and the validity of surgery for the giant pericardial thymic carcinoma.

\section{Background}

Thymic carcinoma is an aggressive epithelial malignant tumour that usually arises in the anterior mediastinum. The intrapericardial thymic carcinoma is rarely occurred case in clinic. It is speculated that it originates from aberrant thymic tissue left behind in pericardium during the embryologic development of thymic gland. Herein, we describe one patient who was diagnosed as giant intrapericardial thymic carcinoma invading superior vena cava (SVC) and right atrium (RA), and underwent mass resection and anatomic reconstruction.

\section{Case Presentation}

A 69-year-old man presented with dizziness and dyspnea. Echocardiography showed a giant mass occupied the pericardial cavity. 2-[18F]-fluoro-2-deoxy-D-glucose $\left({ }^{18} \mathrm{~F}-\mathrm{FDG}\right)$ positron emission tomography/computed tomography (PET/CT) and PET/magnetic resonance imaging (PET/MRI) revealed the soft-tissue mass $(7 \times 4 \mathrm{~cm})$ in the pericardial space with low intensity on T1 weighted imaging and high intensity on T2 weighted imaging and diffusion weighted imaging $(b=800)$ (Supplemental Fig. S1A-C). The mass was FDG avid with the maximum standardized uptake value $\left(S_{\text {max }}\right)$ being 12.4 (Supplemental Fig. S1D). The patient refused surgery and opted for follow-up. Nine months later, the patient presented gradually increased dizziness and polypnea. ${ }^{18} \mathrm{~F}-\mathrm{FDG}$-PET/CT demonstrated increased size $(10 \times 7 \mathrm{~cm})$ and increased ${ }^{18} \mathrm{~F}$-FDG uptake $\left(\mathrm{SUV}_{\max }=17.7\right)(\mathrm{Fig} .1 \mathrm{~A})$. CT angiography (CTA) showed a heterogeneous mass in the pericardial cavity, which was adjacent to SVC, RA and ascending aorta. Meanwhile, massive pericardial effusion was present (Fig. 1B).

Surgical excision of the mass was performed through a median sternotomy. There was no sign of tumour in the anterior mediastinum. The mass was located in the pericardial cavity, adherent to SVC, RA and ascending aorta. Cardiopulmonary bypass was instituted after cannulation of the ascending aorta and inferior vena cava. The mass was carefully separated from ascending aorta. Surgeons performed en bloc 
resection of the mass and a portion of SVC and RA (Fig. 2A). A bovine pericardial patch was used to reconstruct RA and SVC (Fig. 2B). After surgery, the patient felt symptomatic relief and was subsequently referred to oncologists to receive adjuvant therapy.

Hematoxylin-eosin (HE) staining showed the tumour consisted of epithelial and spindle cells, which exhibited clear boundary, prominent nucleoli and highly mitotic figures (Fig. 2C i). Immunohistochemical (IHC) analysis revealed that CD5, CD117 and P40 were highly expressed (Fig. 2C ii-iv). These evidence supported a diagnosis of thymic carcinoma (type $C$ thymoma) that is a high grade malignant epithelial neoplasm.

\section{Discussion And Conclusions}

Thymic carcinoma is a malignant and invasive tumour that easily metastasizes [1]. It usually arises in the anterior mediastinum while few occurs in ectopic locations, including thyroid gland, pericardium, and middle or posterior mediastinum [2]. Very rarely, thymic carcinoma arises intrapericardially. Because the pericardial cavity has limited space, intrapericardial thymic carcinoma may induce mechanical compression or pericardial effusion, as it did in this case. To our knowledge, this was the largest pericardial thymic carcinoma ever to be reported.

Multimodality imaging is necessary for preoperative estimation of mediastinal mass. FDG-PET/CT and FDG-PET/MRI are recommended for management of thymic tumours. A high SUV of 5.0 may differentiate thymic carcinoma from thymoma, thymic hyperplasia, and normal thymus [3]. The mass in this case showed high intensity on T2 weighted imaging, limited diffusion on diffusion weighted imaging, high FDG avidity on PET, and increased size on follow-up, indicating that it was malignant. For thymic tumours, en bloc resection of mass is one of the most important prognostic factors. In this case, CTA provided anatomic information for surgical planning of en bloc resection. Pathological diagnosis of thymic carcinoma was eventually reached. Positivity for CD5 and CD117, in combination with cell morphology, provided strong evidence for the diagnosis of thymic cancer. Also, positive expression of cytokeratin and p63 support the diagnosis of thymic carcinoma [4].

This case demonstrates the utility of multimodality imaging in evaluating the pericardial mass and the validity of surgery for the giant pericardial thymic carcinoma.

\section{Ethics Declarations}

\section{Ethics approval and consent to participate}

This study was approved by the Ethical Committee of Shengjing Hospital of China Medical University. Written informed consent was obtained from individual participant.

\section{Consent for publication}


Written informed consent was obtained from the patient for publication of this case report and any accompanying images.

\section{Availability of data and materials}

The datasets used in the case are available from the corresponding author upon reasonable request.

\section{Competing interests}

The authors declare that they have no competing interests.

\section{Funding}

The manuscript has no funding.

\section{Authors' contributions}

$\mathrm{KZ}$ wrote the manuscript. WC, YZ, HZ and SP revised the manuscript and was involved in pathological analysis. GL is involved in PET diagnosis. KZ, SP and CW are the surgeons of the case. All authors read and approved the final manuscript.

\section{Acknowledgements}

Not applicable.

\section{Abbreviations}

SVC, superior vena cava

RA, right atrium

${ }^{18}$ F-FDG, 2-[18F]-fluoro-2-deoxy-D-glucose

$\mathrm{PET} / \mathrm{CT}$, positron emission tomography/computed tomography

$\mathrm{MRI}$, magnetic resonance imaging

$\mathrm{SUV}_{\text {max }}$ maximum standardized uptake value

\section{References}

1. Engels EA. Epidemiology of thymoma and associated malignancies. J Thorac Oncol. 2010;5:260-5.

2. Weissferdt A, Moran CA. The spectrum of ectopic thymomas. Virchows Arch. 2016;469:245-54.

3. Ferdinand B, Gupta P, Kramer EL. Spectrum of thymic uptake at ${ }^{18} \mathrm{~F}-\mathrm{FDG}$ PET. Radiographics. 2004;24:1611-6. 
4. Marx A, Chan JK, Coindre JM, Detterbeck F, Girard N, Harris NL, et al. The 2015 world health organization classifcation of tumors of the thymus: continuity and changes. J Thorac Oncol. 2015;10:1383-95.

\section{Figures}
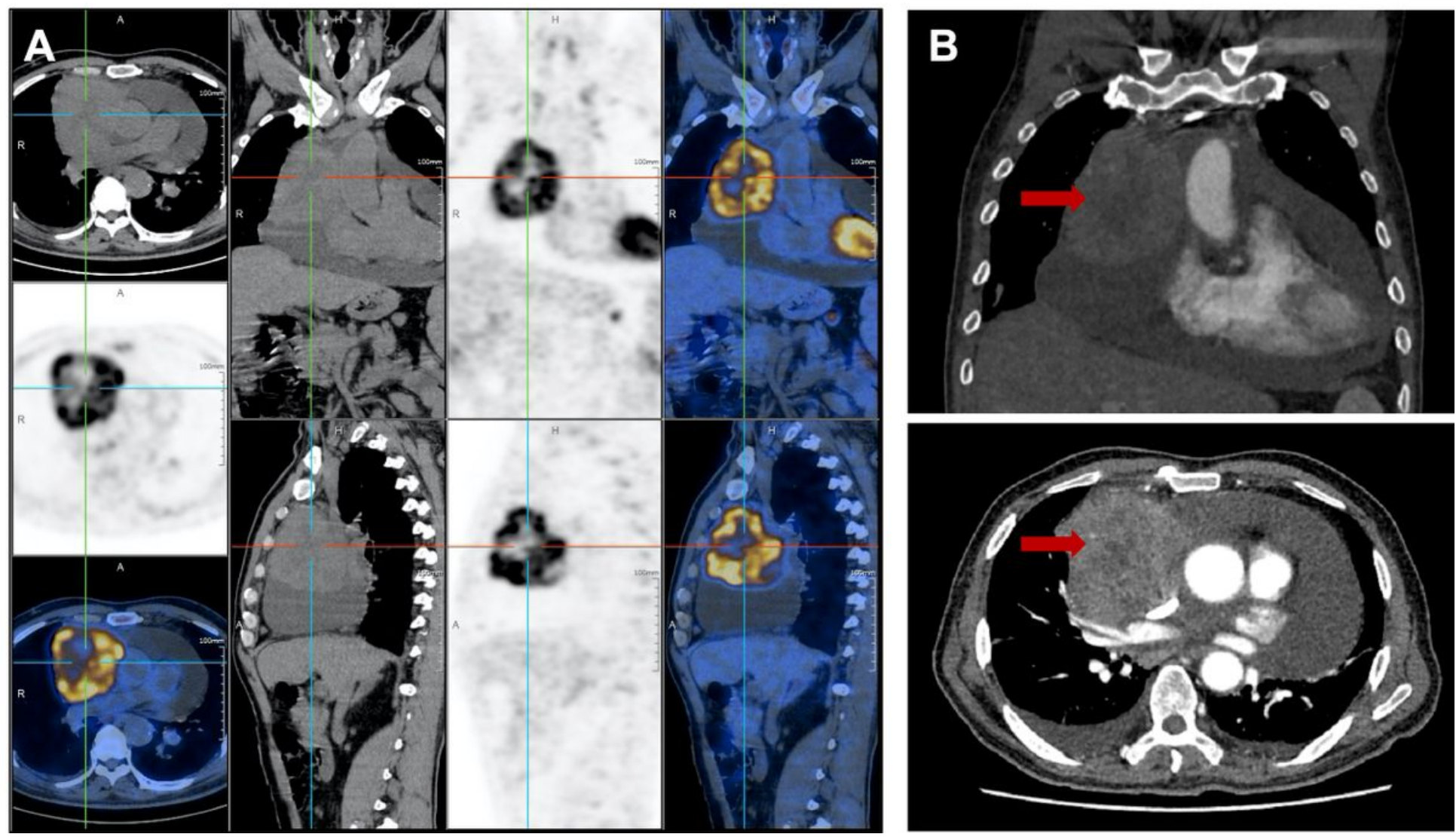

Figure 1

(A) PET/CT showed high avidity in the pericardial cavity. (B) CTA presented a close relationship between the mass and surrounding structures (arrow).
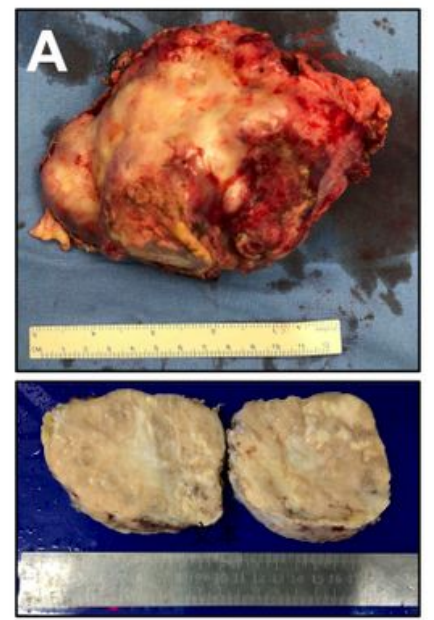

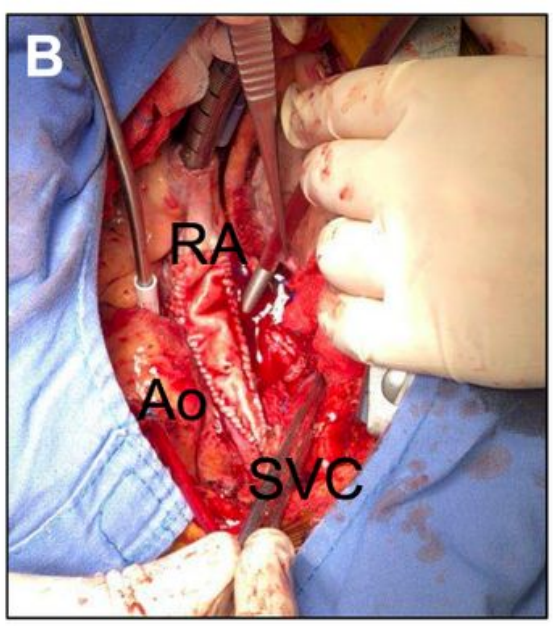

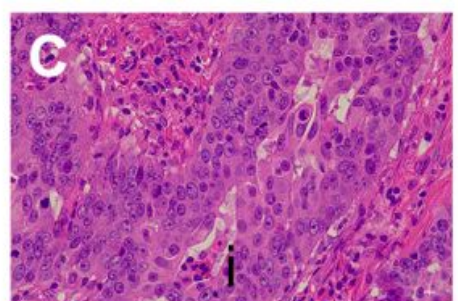

ii

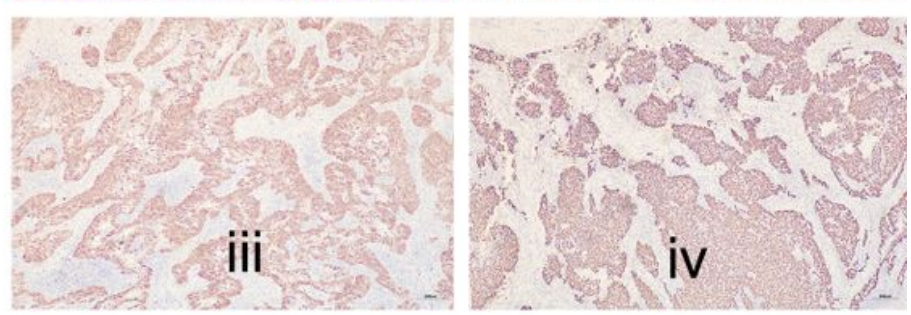


Figure 2

The en bloc excision of the mass was performed (A), and RA and SVC were reconstructed (Ao, aorta) (B). (C) H\&E staining showing the epithelial and spindle tumour cells possessed prominent nucleoli and a high mitotic rate $(\times 20)$ (i). Immunohistochemical staining showed strong expressions of CD5 (ii), CD117 (iii) and p40 (iv) $(\times 20)$.

\section{Supplementary Files}

This is a list of supplementary files associated with this preprint. Click to download.

- CAREchecklist.pdf

- FigS.jpg 